the mother have a rash, fever, adenitis, or arthralgia. A second blood sample was tested in parallel with the first after 10 days. The two showed the same somewhat high level of haemagglutination inhibiting antibodies. ${ }^{2}$ (There was a two-fold reduction in the titre of the first serum after treatment with 2-mercaptoethanol for the removal of IgM antibodies. ${ }^{3}$ ) In the absence of any rise in titre a complement fixation test was done. The result was a two-fold rise in titre-not in itself diagnostic, but the actual level of complement fixing antibodies was relatively high $(1 / 64$ and $1 / 128)$. These result were interpreted to mean that the patient had "recently been infected with the rubella virus." The pregnancy was terminated. Rubella virus was grown from the lung, kidney, and placenta of the products of conception.

When a pregnant woman thought to have been in contact with rubella has no antibodies there is no question of what to do next. When antibodies are found, indicating infection at some time, a problem may arise. This cannot be settled by determining their actual level in a single specimen. There is no absolute figure which is diagnostic of an infection just acquired-not even within a single laboratory using the same method day-by-day, with the utmost care to be consistent. There is variation in the individual immune response to infection and also in the biological materials used in the test for the antibodies which are produced. Therefore the titre of a serum must be considered in the light of the circumstances of the case in order to decide whether the infection may be recent. If so a crisis arises. The case described here shows what may be done to resolve it.-I am, etc.,

T. D. F. MONEY

Public Health Laboratory Service,

Norwich, Norfolk

1 Dudgeon, J. A., British Medical Bulletin, 1969, 2 Stewart, G. L., et al., New England fournal of 3 Banatvala, J. E., Best, J. M., Kennedy, E. A., Medical fournal, 1967, 3, 285 .

\section{Duodenogastric Reflux and Pyloric Surgery}

SIR,-One of the unfortunate aspects of this debate is that pyloric reflux and gastric retention have been proposed as opposing theories in the aetiology of gastric ulcer, whereas they are probably both features of the same underlying pathology. As Mr. $\mathbf{H}$. W. Burge points out (11 November, p. 360), duodenal and pyloric channel disease could give rise to both and, in the presence of a normal pylorus, the antroduodenal motility patterns that are associated with reflux would also tend to delay gastric emptying. The length of time that refluxed duodenal juice remains in the stomach mav determine the degree of mucosal damage. It must also be remembered that there are three types of gastric ulcer, possibly with different causes. ${ }^{2}$

If the gastric ulcer is clearly secondary to duodenal or pyloric disease (Type II) and the acid secretion is moderate or high, then vagotomy is theoretically a reasonable operation to heal the duodenal ulcer. But ulcers cause scarring when they heal and a scarred duodenum and pylorus may perpetuate a delay in gastric emptying and pyloronlasty may be required in addition.

With the primary lesser curve ulcer (type I), for those who suggest that the main problem is increased antral gastrin release following gastric stasis the two logical alternatives are a Billroth $I$ antrectomy or an adequate simple drainage operation, but the latter has not proved effective. We would suggest that a proximal gastric vagotom without drainage does not follow logically from the hypothesis of gastric stasis in patients without associated duodenal or pyloric disease. For those who think that reflux is the primary problem the logica operation is a Roux-en-Y reconstruction (with vagotomy to protect the jejunum). The alternatives are: $(a)$ an operation that allows little reflux, and this is probably provided by a Billroth I antrectomy with a smal stoma; or (b) an operation that allows rapid emptying once reflux has occurred, which may be provided by a wide pyloroplasty, but this also gives rise to increased reflux.

If we look at the operations themselves, we find that the great success of the Billroth $\mathrm{I}^{3}$ operation would support both hypotheses, perhaps favouring the antral gastrin release theory in particular. The moderate success of vagotomy and pyloroplasty ${ }^{3}$ also adds some support to both hypotheses, perhaps by overcoming stasis and allowing quick drainage of refluxed duodenal juice, but it is not certain how the vagotomy helps if the night and stimulated gastric acid secretions are already very low. Proximal gastric vagotomy without drainage is logical only if it is thought that a small duodenal or pyloric lesion will heal without scarring and pyloric function and gastric emptying will return to normal.

It is too early in our understanding to be over-dogmatic about one or other aetiological theory or method of rreatment, but we should try to make our surgical practice consistent with our theories of the pathophysiology of gastric ulcer and then continually adjust our hypotheses on the basis of a careful analysis of the results of treatment.-We are, etc.,

A. G. Johnson K. W. REYNOLDS

Charing Cross Hospital Medical School,

Fulham Hospital

1 Johnson, A. G., British fournal of Surgery, 1971, 58,864

Johnson, H. D., Annals of Surgery, 1965, 162,

Duthie, H. L., Gut, 1970, 11, 540.

\section{Hypotension and Methylmethacrylate Cement}

SIR,-We have read with great interest the various communications on this subject which have appeared during the past 12 months. ${ }^{1-7}$ It would seem that there is still continuing concern regarding the use of acrylic cement in the fixation of prostheses in major hip arthroplasty. Having been responsible during the past 10 years for either the personal administration or supervision of anaesthesia for more than 7,000 hip arthroplasties performed at this centre in which acrylic cement has been used, we feel it may be of some value to present certain of our findings based on this experience.

(1) A fall in blood pressure does not invariably follow the introduction of acrylic cement into either the newly reamed acetabulum or femoral shaft, although it is a common occurrence ( $80 \%$ of cases approximately).

(2) When a fall in blood pressure occurs following insertion of cement into the reamed acetabulum it is small and transient, rarely exceeding $15 \mathrm{~mm} \mathrm{Hg}$.

(3) Following insertion of cement into the reamed femoral shaft the fall in blood pressure is usually greater, but rarely exceeds $30 \mathrm{~mm} \mathrm{Hg}$. The time taken for a maximum fall to occur is usually $30-60$ seconds and there then follows a rapid return to normal, which seldom takes more than a further 90 seconds. We have not noticed the late rise in pressure recorded by Cadle et al.

(4) In no instance at this centre has cardiac arrest occurred following the use of acrylic cement, nor have falls in blood pressure given regular cause for concern. Neither are we aware of any patient who has sustained untoward sequelae as a result of the use of the cement. Many of our patients are elderly and frail, and hypertension and coronary artery disease are only too common. Such patients, we agree, compensate poorly following sudden and severe hypotensive episodes, but in our experience the use of acrylic cement does not produce the changes in pulse rate and hypotension which were shown to follow the intravenous injection of monomer into dogs by Peebles et al. ${ }^{2}$ This is probably not surprising, as the absorption of monomer into the circulation from reamed bone must be considerably slower and blood levels thus proportionately lower. Continuous electrocardiographic tracings carried out on a large number of our patients have never shown any change in character following the use of acrylic cement.

(5) There seems to be conclusive evidence $^{2} 4$ that hypotension following the use of acrylic cement is in fact due to the absorption of free monomer into the circulation, and it is interesting to note in this connexion that in our experience no fall in blood pressure follows the use of cement in a knee arthroplasty performed under a tourniquet, a fact already noted by Cole et al. ${ }^{7}$ From our observations we are convinced that a careful surgical appraisal of the degree of plasticity of the cement prior to its insertion, preceded by a careful technique of preparation, are the important factors which really reduce subsequent hypotension to a minimum. Any departure from this established technique ${ }^{6}$ which entails insertion of cement in a more fluid state, particularly into the femoral shaft, will undoubtedly cause a greater fall in blood pressure.

(6) It is not our experience that hypotension following the use of acrylic cement is in any way modified by the choice of a particular type of anaesthetic technique. Over the past 10 years anaesthesia for hip arthroplasty here has embraced all types and combinations of anaesthetic agent, including neuroleptanalgesia, extradural analgesia, and induced hypotensive techniques. Halothane is currently used in combination with other agents in approximately $70 \%$ of cases. Nor does the type of operation carried out appear to be significant. A patient undergoing a single uncomplicated hip arthroplasty does not differ in his response to cement from the patient undergoing a bilateral arthroplasty taking up to $3 \frac{1}{2}$ hours with a proportionately higher blood loss. Technically difficult conversion arthroplasties, in which there may be extensive reaming of the femoral shaft and relatively higher blood loss, have shown surprisingly little difference in response to cement, though theoretically a greater fall in pressure should be expected in them.

Although we would agree that there is no place for complacencys we have not 